\title{
Modelos de gestión de la innovación docente con uso de TIC 1 .
}

\author{
Teaching innovation management models with ICT use.
}

\author{
Eduardo Augusto Duque Cuesta ${ }^{2}$ \\ Jorge Luis Rojas García ${ }^{3}$ \\ Yogel Alberto Ruas Amaya ${ }^{4}$ \\ José Francisco Amador Montaño 5 \\ Héctor Gerardo Sánchez ${ }^{6}$
}

Duque Q. Eduardo A, Rojas G. Jorge L. Ruas A.Yogel A. Amador M. José F. Sánchez. Héctor G. miradas $\mathrm{N}^{\circ} 13$ - 2015. ISSN: 0122 994X Págs 27 - 40

Recepción: Mayo 15 de 2015

Aprobación: Octubre 6 de 2015

Publicación: Diciembre 17 de 2015

\section{Resumen}

El presente artículos es de investigación, su objeto de estudio fue la identificación de los modelos de gestión de las prácticas educativas innovadoras en las cuales los docentes hacen uso de las TIC para impactar los proyectos de aula en la escuela, estos docentes usaron la metodología de ayudas hipermediales dinámicas (AHD). La investigación de tipo descriptiva con diseño no experimental y enfoque cuantitativo, los datos se recolectaron vía web usando el software Limesurvey, para la captura datos en línea; estos datos se procesaron en SPSS y entre los resultados más relevantes se encontró una tendencia predominante entre los docentes hacia el modelo de iniciación de Salinas (2004) o llanero solitario de Bates (2012). Como recomendaciones especiales, el documento tiene algunas sugerencias sobre la gestión administrativa, tecnológica y pedagógica que dan orientaciones para que las Instituciones educativas puedan favorecer la innovación docente con las TIC, entre ellas que deben establecerse planes estratégicos de las TIC en 1 Proyecto Computadores para Educar MinTIC. Región 3. Gerencia el proyecto UTP. Grupos de Investigación CRIE y TICER.

2 Candidato a Doctor en Educación, Magister en Comunicación Educativa, Esp. Gerencia de Tecnologías, Esp. Informática y Telemática. Docente Facultad de ciencias de la Educación y Facultad de Ingeniería Industrial UTP. Director Departamento de Informática Educativa Fundación Universitaria del Área Andina. eduque@areandina.edu.co

3 Magister en Comunicación Educativa, Esp. Gerencia de Tecnologías. Gerente del proyecto Computadores Para Educar región3.jorojas@utp.edu.co

4 Candidato a Doctor en Educación, Magister en Informática Educativa, Esp. Informática y Telemática. Docente Facultad de Ingenierías y Ciencias Básicas en el Departamento de Informática Educativa Fundación Universitaria del Área Andina. yruas@areandina.edu.co

5 Magister en tecnologías de la información aplicadas a la educación, Especialista en gerencia y gestión cultural. Docente Facultad de Ciencias de la Educación UTP, Coordinador académico proyecto CPE Región 3. jamador@utp.edu.co

6 Candidato a Doctor en Educación, Magister en Comunicación Educativa, Esp. Gerencia de Tecnologías, Esp. Computación para la docencia. Docente Facultad de ciencias de la Educación UTP y Colegio INEM Felipe Pérez. hgsanche@utp.edu.co 
la Institución, los cuales también ayudan a una mejor educación con calidad.

Palabras Clave: TIC, Educación, Gestión de la innovación, AHD, Gestión educativa, innovación educativa.

\section{Abstract}

This article is about research, your object of study is the identification of management models for innovative educational practices in which teachers make use of ICT to impact classroom projects at school, these teachers used the methodology called dynamic hypermedia aid (DHA). The research is descriptive with non-experimental design and quantitative approach, data were collected using the LimeSurvey software for online data capture; these data were processed in SPSS and, among the most relevant results, a predominant tendency through teachers was to the initiation model of Salinas (2004) and lone ranger Bates (2012). As special recommendations, the document has some suggestions on administrative, technological and pedagogical management that give guidance for educational institutions to promote educational innovation with ICT, including ICT strategic plans to be established in the institution, which also help to a better quality in education.

Key Words: ICT, Education, Innovation Management, education Management.

\section{Introducción}

El presente trabajo de investigación se realizó con docentes participantes del programa Computadores Para Educar (CPE) que fueron finalistas en el Vitual Educa Regional 2014, en Pereira, este evento educativo cubrió la región 3 de CPE, que está comprendida por los departamentos de Caldas, Quindío,
Risaralda y Valle del Cauca, con Recursos educativos realizados bajo la metodología de Ayudas Hipermediales dinámicas (AHD). Estos logros de estos profesores se consideran exitosos, puesto que fueron seleccionados para representar sus regiones por mostrar un avance significativo en su desarrollo y aplicación en el aula, es decir, pedagógica y técnicamente se consideran bien construidos, es por ello, que estos docentes contaron, mediante las encuestas que respondieron en esta investigación, que tanto le aportó la gestión administrativa, la gestión tecnológica y la pedagógica al modelo de gestión que ellos usaron para innovar educativamente con las TIC en el aula.

El objetivo principal esta investigación consistió en buscar aquellos elementos comunes entre los docentes innovadores $\mathrm{y}$ exitosos, de manera que puedan ser replicados en otros, ser implementados en instituciones educativas y aprovechar de mejor manera la dotación tecnológica que el gobierno está entregando en las escuelas del país, logrando impactar de manera positiva la educación con el uso de las TIC. Un docente innovador no sólo mejora las condiciones educativas, también motiva a los estudiantes y esto disminuye el ausentismo escolar y mejora los indicadores de aprendizaje.

Para lograr determinar esos elementos comunes que favorecen la innovación educativa con las TIC, la investigación se basó en un marco teórico con autores internacionales y nacionales, que entre ellos logran una fusión que otras investigaciones no habían hecho, ya que casi todas se basan en lo tecnológico o en lo pedagógico; en esta oportunidad, no sólo se fusionan estos dos tipos de gestiones (Tecnológica y pedagógica), se le adiciona la gestión administrativa, para lograr unos modelos completos, en los cuales la gestión 
del directivo de la institución educativa tiene una influencia muy marcada en los logros de sus docentes, dado que puede, mediante políticas institucionales ofrecer un ambiente que favorezca el uso de las TIC en la educación.

Para lograr esta fusión y determinar los elementos que se debían indagar entre los docentes, no sólo bastó encontrar autores de la talla de Tony Bates, Albert Sangrá, Jesús Salinas, Cesar Coll, estudios y publicaciones de la UNESCO y muchos más, también se recurrió a un diseño metodológico basado en la investigación descriptiva con enfoque cuantitativo, de diseño no experimental para determinar matemáticamente la ubicación del modelo de gestión de la innovación docente con las TIC que impera en la región estudiada.

Todo este trabajo se realizó entre los docentes que ganaron su participación en el Educa Digital Regional de 2014, efectuado en Pereira, Risaralda, que llegaron a este evento por ser considerados exitosos, primero, en sus Instituciones educativas, ya en representación de su Institución educativa, participaron a nivel departamental y luego a nivel regional, según los puntajes de los jurados, se obtuvo entre los 4 departamentos de la región 3 de CPE (Caldas, Quindío, Risaralda y Valle del Cauca) un total de 70 participantes, los cuales debían, según el área de aprendizaje, presentar su trabajo ante un público conformado por aproximadamente 500 docentes e invitados de las secretarías de educación de la región citada y ganarse uno de los 10 cupos al evento nacional de CPE en Bogotá. La duración de esta investigación fue cercana a un año, durante ella se dieron los eventos descritos en todo el proceso.

\section{Marco Metodológico}

\section{Tipo de Investigación}

Esta investigación no intenta dar explicación respecto del problema del uso de las TIC de los docentes en la región, lo que realmente buscó fue recoger e identificar antecedentes generales, tendencias, temas y tópicos respecto al uso exitoso de las TIC en el aula de clases, por consiguiente se hacen sugerencias de aspectos relacionados que deberían examinarse no sólo en futuras investigaciones, sino en toda institución educativa que quiera tener éxito al implementar las TIC en sus proyectos de aula. El objetivo es documentar en el campo si los docentes se pueden identificar con un modelo para gestionar la innovación educativa con las TIC en los proyectos de aula usando las AHD (Rojas et al. 2013), tal y como lo contempla la propuesta de CPE, en la cual todos los docentes seleccionados las usan.

Por todo lo anterior esta investigación es de tipo descriptiva, con diseño no experimental y enfoque cuantitativo, ya que se acomoda a todas las necesidades anotadas. En este tipo de investigación por lo general se investigan tendencias, por ello, se buscó especificar las propiedades relevantes de personas, grupos o comunidades que están sometidas al estudio, para medir y evaluar los aspectos relacionados con el fenómeno que se está investigando y lograr determinar la ubicación del modelo general de innovación educativa con las TIC presente en los docentes de la región 3 de CPE.

\section{Metodología de la Investigación:}

La metodología fue de enfoque cuantitativo, se usó la estadística y los resultados arrojados por el software estadístico SPSS para determinar las frecuencias, tendencias y otras medidas estadísticas, todo basado 
en las respuestas de los docentes que hacen parte de la población estudiada y contestaron las encuestas, adicional a esto, se revisaron los trabajos de los docentes, las evaluaciones de los jurados y la socialización con el equipo CPE y los directivos docentes que acompañaron este proceso, en el cual el centro de la investigación se basa en las repuestas de los docentes considerados innovadores.

Para que un docente logre la innovación educativa en el aula haciendo uso de las TIC, se trabajaron con las categorías o modelos propuestos por Salinas (2004), pero se soportan en otros investigadores como Tony Bates (2004) y (2012), Cesar Coll (2007), Rojas et al. (2013), Duque \& Rojas (2012) y otros, los cuales contemplan sus propias categorías o modelos, todas ellas muy orientadas al trabajo del docente, por lo tanto, lo que se trata de hacer en este trabajo es ampliarlas al modelo de gestión que predomina entre los docentes, al contexto tecnológico, incluyendo apropiación y su contexto pedagógico.

Estas categorías son:

- Gestión Administrativa

- Gestión tecnológica y

- Gestión Pedagógica

\section{Gestión Administrativa:}

La primera categoría tienen que ver con la gestión de las directivas institucionales y su visión de uso de las TIC para impactar positivamente la educación escolar, por lo que se ha denominado Gestión Administrativa, la cual aplica de manera especial al trabajo que realizan las directivas escolares, no sólo en dotación y mantenimiento de la infraestructura física tecnológica de la institución educativa, es también articular su PEI, planes estratégicos alienados con la misión, la visión y objetivos estratégicos con los planes estratégicos TIC, no sólo para favorecer el uso pedagógica de las TIC, sino para impactar positivamente la calidad educativa con estas tecnologías.

En esta categoría, las directivas académicas deben tener definido un plan estratégico de TIC articulado con el PEI y el PMI (Plan de mejoramiento Institucional, Guía 34 MEN), también deben establecer cuáles son las prioridades, estrategias y políticas Institucionales a impactar con las TIC, procesos de formación, actividades y hasta indicadores que puedan, en determinado momento, servir para el monitoreo y determinar si se va por buen camino o no y los posibles ajustes que se deben hacer para logar la visión a la cual la apunta la institución educativa.

En esta categoría, pueden influir en el modelo para la gestión de la innovación educativa las siguientes variables:

Según Bates (2004), la Institución Educativa debe tener un plan para gestionar la adquisición, uso y apropiación de las TIC, cuyo líder debe ser la máxima autoridad Institucional.

Según Salinas (2004), las Instituciones Educativas tienen mucho más, normalmente, de lo que requieren para innovar, para ello deben tener una infraestructura técnica y materiales de apoyo para soportar las necesidades del docente innovador.

\section{Gestión Tecnológica:}

La segunda categoría es de carácter técnico, en especial la manera como las directivas académicas visualizan la tecnología y su apropiación como apoyo a los procesos de enseñanza y aprendizaje y como la infraestructura técnica debe estar 
en óptimas condiciones de modernidad, capacidad y cantidad adecuada; esta categoría tiene que ver con la dotación que tiene la Institución Educativa en equipos, conectividad, plataforma de apoyo a la educación como CMS, LMS, LCMS y otras; que tan sincronizado está el parque tecnológico de la Institución respecto al deber ser que descrito en el PEI o en el plan institucional o estratégico de TIC (Temáticas o PlanTIC), debe existir un proceso de apropiación tecnológica donde se articule lo cultural, organizacional y técnico de la tecnología con el que hacer docente en el aula de clases. También debe haber un grupo de estudiantes debidamente preparados con conceptos básicos en uso del computador y de ser posible, una persona responsable de toda la infraestructura técnica de la institución (Salinas, 2004), (Bates, 2004) el coordinador TIC de la institución.

\section{Gestión pedagógica:}

Esta categoría va más allá de un uso instrumental del computador, de hecho, en esta se hace una reflexión sobre que se enseña, que se aprende y que y como se evalúa; adicionalmente, que motiva al estudiante a aprender, en especial usando las tecnologías, ello se logra mediante procesos de formación en estrategias pedagógicas y didácticas con el uso de tecnologías en el aula (Coll, 2007) y (Rojas et al., 2013). Estos procesos de formación deben ser continuos, es decir, si la tecnología cambia cada año y se renueva cada tres años, los docentes deben estar en permanente formación y actualización, lo cual hará que la innovación en estos, los docentes, se convierta en parte de su vida, innovar no debe ser una alternativa en un punto de tiempo, debe ser una forma de vida y cada vez que un docente pone en prueba un nuevo diseño, ya está pensando cómo mejorarlo o adecuarlo para que cumpla de mejor manera su función, ya sea usando otra aplicación, lenguaje o lo que haya usado para generar su AHD.

\section{Resultados}

Ahora se describirán los resultados arrojados por el procesamiento de la información, resultado del DATA obtenido de SPSS una vez procesados los resultados de la encuesta aplicada, esto permitió establecer relaciones y teorías claves acerca de cómo los docentes de la región han innovado con las TIC en sus prácticas educativas, los cuales trabajaron en el modelo de formación de CPE en la región 3 con ayudas hipermediales dinámicas (AHD) con enfoque socio constructivista, identificando en las didácticas usadas los modelos de gestión administrativa, tecnológica y pedagógica.

\section{Preguntas sin categoría:}

El 52,5\% de los docentes son del área de tecnologías y el $17,5 \%$ son del área de ciencias, las demás áreas se encuentran mínimamente representadas entre los mejores trabajos. Vale la pena citar que el proceso de formación de CPE docentes de todas las áreas son incluidas, pero definitivamente el área de mayor representatividad es la de tecnologías, adicionalmente, contrario a lo que se supondría, los docentes con mayor participación son los que tienen más de 7 años de experiencia, estos docentes tienen participaciones más representativas que los docentes que tienen pocos años y alcanza más del $60 \%$ de los docentes. Así mismo, se encontró que también al menos el $60 \%$ de los docentes lleva menos de 4 años haciendo uso de las TIC, es decir, de alguna manera ese porcentaje alto de los docentes que tuvieron trabajos considerados exitosos, tan sólo desde hace 4 años vienen trabajando con las TIC para impactar su 
currículo, el $20 \%$ del total de los docentes sólo ha trabajado con las TIC el último año, en el marco del proyecto de formación de CPE, lo cual debe considerarse un éxito.

\section{Gestión administrativa:}

Las respuestas de los docentes sobre esta categoría, la cual tiene que ver con la gestión de las directivas institucionales y su estrategia para el uso y apropiación de las TIC para impactar la educación. Esta categoría aplica de manera especial al trabajo que realizan las directivas escolares, no sólo en dotación y mantenimiento de la infraestructura tecnológica, es también tener en el PEI y en un Plan Institucional de TIC (Temáticas) definidas la visión, prioridades, estrategias y políticas Institucionales a realizar con las TIC, procesos de formación, actividades y hasta indicadores que puedan en determinado momento servir para el monitoreo y determinar si se va por buen camino o no y los posibles ajustes que se deben hacer para logar la visión a la cual la apunta la Institución Educativa.

Los docentes encuestados, los cuales fueron representantes de sus Instituciones educativas por la calidad, cualidad e impacto educativo de sus trabajos consideraron que el $42,50 \%$ de las Instituciones educativas tiene un plan liderado por las directivas, el cual ha permitido que realicen procesos de capacitación y haya adquisición de equipos acorde a ese plan y la formación de docentes es fundamental en él. Mientras que el $27.50 \%$ de los docentes dicen que su institución no tiene un plan, es decir, carece de una carta de navegación para la adquisición tecnológica y la formación docente en ello, estas instituciones, dependen de las políticas externas a ella sobre este aspecto, es decir, MINTIC, MEN, Gobernación, Alcaldías. Referente a esta gestión, se asegura que todas las instituciones educativas oficiales tienen en plan desarrollado mediante el programa TemaTICas del MEN, sin embargo, menos de la mitad de los docentes dice que existe, a este respecto, Bates (2004) y Bates \& Sangrá (2012) infieren muy importante no sólo tener establecido un plan con metas e indicadores a cumplir, también creen que el socializarlo y presentar de manera periódica sus logros es necesario para la adecuada apropiación de la comunidad y su participación en este

En cuanto al número de equipos en las Instituciones educativas, el $37,5 \%$ considera que hay pocos equipos, pero que algo se puede hacer con ellos, piensan que se deben tener más, mientras que el $27,5 \%$ supone que hay suficientes equipos para hacer un buen trabajo y piensa que el problema es el acceso a ellos pues su uso es restringido o mantienen en mal estado. Pero hay un número también representativo de $32.5 \%$ de estos docentes que creen hay un buen número de equipos en excelente estado y manera de acceder a ellos cada vez que lo requieran. El acceso a los computadores por docentes y estudiantes es un elemento esencial para que haya innovación educativa con las TIC, Salinas (2004). “... el papel de las autoridades educativas en el tema del equipamiento y de la orientación del proceso, el acceso a fuentes de información adecuadas" (Salinas, 2008, p.129), deben ser el primer punto para que haya innovación en el aula con las TIC, ya que garantiza la disponibilidad del recurso informático para el docente y el estudiante (Bates, 2004)

El $45.00 \%$ de los docentes consideran que la Institución Educativa o sede no ha tenido el apoyo de la administración municipal o departamental para dotarla, pero salió favorecida por Computadores para Educar y por ello tiene algunos equipos. Otro porcentaje de docentes, no tan alto como 
el anterior, piensa que la Administración Municipal y las directivas de la Institución se han tomado la tarea de solicitar equipos para la Institución, pero no han logrado que tengan los suficientes. Otro porcentaje más representativo, $30,00 \%$, opina que las directivas Institucionales apoyadas con la administración Municipal y/o Departamental han realizado una gestión extraordinaria y han logrado conseguir una excelente dotación de equipos y siguen gestionando para su adecuada actualización. Se evidencia que los entes territoriales hacen un esfuerzo en dotación de tecnologías, el indicador de niños por computador va disminuyendo, la meta para el 2014 era de 12, lo cual se cumplió en el año 2013 (www.mintic.gov.co). La planificación para la apropiación de las TIC en la institución educativa, su dotación y renovación permanente debe estar no sólo escrito, debe tener un indicador, por ejemplo, renovación cada tres o cuatro años, para garantizar la no obsolescencia tecnológica de las salas de informática (Bates \& Sangrá, 2012)

Sólo el 5\% de los docentes encuestados consideran que el PEI no sólo contempla a las TIC en varias partes sino que le dedica una sección de este documentos para hacer una reflexión sobre el potencial educativo de la tecnología, mientras que un $27.50 \%$ sólo lo cita en uno de sus apartes, el $47.50 \%$ reflexiona que lo cita en varias partes y adicional lo ve como una estrategia de formación y calidad académica para mejorar los procesos de enseñanza y aprendizaje. "El pensamiento estratégico y su construcción y comunicación a través del proceso de planificación más o menos formal, ayuda mucho a conseguir que la tecnología se integre bien en una institución" (Bates \& Sangrá, 2012, p.123). Este pensamiento estratégico debe estar plasmado en un documento, el cual muchas instituciones educativas tienen pero la mayoría no aplica y en algunos casos no es conocido por su comunidad.

El $45 \%$ de los docentes creen que la Institución Educativa está pendiente de ellos, piensa que estos realizan actividades interesantes, por este motivo, logran apoyos y permisos de las directivas para salir en su representación en los eventos regionales y nacionales, mientras que un $32.50 \%$ supone que en la Institución le prestan atención sólo para aquellos exitosos con las TIC, cuando hacen algo interesante y salen a representarla, sin embargo, hay que implorar para un permiso o apoyo. La Institución Educativa debe tener elementos para la motivación de sus profesores y llevarlos hacia la innovación educativa, estos elementos vas desde el apoyo con estudiantes avanzados, el acceso a espacios dotados con tecnologías (laboratorios o aulas con tecnologías) hasta brindarle espacios de tiempo y apoyo para asistir a procesos de formación, congresos y seminarios donde no sólo vea logros y experiencias de otros docentes, sino que exponga las propias en representación de la Institución (Bates, 2004), (Bates \& Sangrá, 2012). Es por ello que la institución debe motivarlos, especialmente a los inquietos y los apoyarlos con los recursos que tiene a su disposición (Salinas, 2008).

En cuanto a equipos de trabajo constituidos formalmente y apoyados por las directivas escolares, sólo el 7\% piensa se hace, el resto, los mismos docentes por iniciativa propia, buscan espacios y se reúnen a trabajar y apoyarse mutuamente con las TIC $(47.50 \%)$ y un grupo menos representativo con el $35 \%$ cree que el docente de TIC los apoya y tiene el beneplácito de las directivas escolares, pero lo hacen en su tiempo libre, esto no favorece la innovación educativa con TIC, Bates (2004) y (Bates \& Sangrá, 2012) afirman que uno de los modelos para innovar con las TIC consiste en el trabajo 
en equipos, donde el docente comparte experiencias y recursos con sus colegas aunando esfuerzos en el desarrollo de materiales "Colegiados" que serán usados con sus estudiantes (Salinas, 2004).

Sólo el 5\% de los encuestados supone que existe una articulación, para la inversión en tecnologías, entre el PEI y el plan TIC (temáticas) y que estas inversiones obedecen a lo descrito en ellos, sin embargo, el $42,50 \%$ admite que eso depende de los entre territoriales o nacionales como MIN TIC, que muchas veces lo planteado en el PEI es complejo hacerlo cumplir. Existen casos en que sin pretenderlo, el costo que asume la Institución o entes gubernamentales, con la inversión en tecnologías, la terminan asumiendo otros grupos, como docentes con mayor cantidad de trabajo, recortes en otras áreas, en fin, consecuencias no deseadas, es por ello, que tener claro cómo se debe hacer la inversión y que esta obedezca a un plan trazado institucionalmente, hace que; primero, se concentren los recursos en lo que es importante y segundo, que no se sacrifiquen otras áreas por improvisación en el uso de las inversiones (Bates \& Sangrá, 2012).

En cuanto a un plan de formación docente articulado por la Institución educativa y escrito en sus documentos Institucionales como el PEI o el plan estratégico de TIC (Temáticas) sólo el 12,5\% razona que este existe y es liderado por el rector, el resto especula que no existe tal plan el $37.50 \%$, que es contemplado en estos documentos pero no es operativo o funciona a medias el $47.50 \%$. En consideración, se asegura que “...la formación del profesorado en el terreno de las TICs, debe superar con creces la simple capacitación para el manejo instrumental, y alcanzar otras dimensiones más significativas desde la metodológica, hasta la de investigación" (Salinas, 2008, p. 95).
Ninguno de los encuestados conoce que la Institución educativa tenga un equipo que lidere y apoye a los docentes en el uso de TIC en el aula, sin embargo, un 30\% de los ellos, infirió que en sus Instituciones existe un grupo de docentes entusiastas que cuentan con el beneplácito de los directivos y un $60 \%$ razona que deben hacerlo por iniciativa propia. "El trabajo en equipo es fundamental, pero a diferencia del aprendizaje grupal, en el aprendizaje colaborativo no existe un líder, sino que cada uno actúa como líder en la tarea que le ha designado el grupo" (Duque \& Rojas, 2012, p. 49). Es entonces cuando adquiere valor relevante el trabajo colaborativo entre los profesores, los cuales interactúan entre áreas similares o cursos iguales, con el objeto de compartir experiencias y hacer una construcción colectiva que les sirva a todos (Bates \& Sangrá, 2012).

\section{Gestión Tecnológica:}

La segunda categoría es de carácter técnico, en esta, los docentes respondieron sobre el potencial de la tecnología, los recursos que se poseen, como se administran, si son suficientes en cantidad y calidad y la infraestructura tecnológica institucional como se encuentra (Bates, 2004). Si existe un proceso de apropiación tecnológica donde se articule lo cultural, organizacional y técnico de la tecnología con el que saber hacer docente en el aula de clases. Debe existir un grupo de estudiantes debidamente preparados con conceptos básicos en uso del computador y de ser posible, una persona responsable de toda la infraestructura técnica de la institución.

Un $7.5 \%$ de los docentes encuestados cree que existe un inventario de equipos debidamente socializado y que también conocen los indicadores internos y los nacionales de estudiantes por computador, tienen claro en qué situación se encuentran. Mientras que existe un $62,50 \%$ que 
presumen que hay un inventario, el cual pocos conocen, pero no tienen idea de indicadores y como se encuentra la Institución con respecto al departamento o la nación. Si bien, lo ideal es que todos en una institución educativa conozcan los recursos que tienen a disposición, (Bates, 2004), Una de las condiciones que favorece la innovación educativa según Bates \& Sangrá (2012) es conocer con lo que se cuenta en la institución educativa, muchas veces se ignora con qué recursos se cuenta, muchas instituciones tienen tableros digitales y equipos sofisticados guardados, pues no los saben utilizar; el dar a conocer el inventario con el cual se cuenta puede estimular a los docentes a innovar y probar nuevas estrategias que pueden abrir otros caminos.

Más del $80 \%$ de los estudiantes tienen competencias básicas o avanzadas en uso de computadores, por lo tanto, puede ser propio pensar en semilleros que de alguna manera apoyen y articulen la estrategia de la Institución educativa, ya que los estudiantes no tienen que aprender sobre uso de los equipos, por ello, deben los docentes es enfocarse en el uso pedagógicos en sus asignaturas y que las TIC sean un estímulo para los estudiantes.

Un estudio realizado en Iberoamérica, por la fundación Telefónica, se considera que "...los menores en los países iberoamericanos están creciendo en un contexto altamente tecnologizado, lo que plantea nuevas oportunidades y nuevos retos desde el punto de vista educativo y de la protección del menor" (Bringué, Sádaba, \& Tolsá-Caballero, 2011, p.13). En el citado estudio de Telefónica, sobre el acceso a computadores, MP3, reproductores de discos ópticos (DVD, Bluray y otros), se asegura que "...cabe la pena reseñar también que mientras el 9\% de los menores de 6 a 9 años declaran no poseer ninguno de estos dispositivos" (Bringué, Sádaba, \& Tolsá-Caballero, 2011 , p. 29) lo que significa que más del $90 \%$ de estos menores tiene y mantiene contacto con las nuevas tecnologías, lo cual significa un reto para sus docentes. En el contexto colombiano, el Ministerio de las TIC está dotando con tabletas las instituciones educativas oficiales y algunas de ellas tienen esquema de trabajo 1-1 (un estudiante por computador).

El 27,50\% de los docentes opina que la Institución educativa hace un esfuerzo y destina recursos a los procesos de formación, está pendiente de las convocatorias y siempre abre espacios para que sus docentes se beneficie, facilitándoles el lugar y tiempo para hacerlo, mientras que hay un $65 \%$ que cree no apropia recursos así quisiera, siempre están pendientes de no afectar el horario laboral y en algunos casos busca reemplazos para no afectar a los estudiantes, pero no es una prioridad. "...no es suficiente con dotar a las escuelas con computadoras o con acceso a Internet: también es necesario trabajar en la formación docente y en la formulación de nuevos repertorios de prácticas que permitan hacer usos más complejos y significativos de los medios digitales" (Dussel, 2011, p. 12).

El $12,50 \%$ de los docentes dice que la Institución posee una plataforma LMS (Learning Managment System - Sistema de Administración de Aprendizaje) para administrar sus contenidos educativos, un $20 \%$ afirma que tienen acceso a una del ente territorial desde la Secretaría de Educación, el resto, no tienen, pero un buen porcentaje tiene en sus planes usar una en el futuro. "Los usos que los participantes hagan efectivamente de las TIC, dependerán en buena medida, de la naturaleza y características del equipamiento y los recursos tecnológicos puestos a su disposición" (Coll, 2008, 
p. 86) al referirse a participantes Coll, se refiere a los estudiantes y cómo las plataformas LMS pueden aportar al trabajo en clase y fuera de esta. Así mismo, Bates y Sangrá (2012), consideran que tener acceso a un sistema de administración de aprendizaje ayuda a los estudiantes a ser más responsables a tener una ruta de aprendizaje y a realizar autogestión de los contenidos educativos, es un gran apoyo para la educación presencial y preparar para al aprendizaje autónomo.

Los docentes responden que sólo el 7,50\% de ellos conocen que en su Institución haya manejo de indicadores de uso de los recursos tecnológicos, un $47,50 \%$ cree que sólo tienen manejo de horarios y de estos obtienen niveles de uso, el resto que no conoce de indicadores ni otro tipo de datos o estadísticas que indique la usabilidad de su dotación de tecnología o tiempo ocioso de la misma. Los indicadores son una buena forma de estar verificando que se hace con la dotación de tecnologías y en que se está usando. Bates y Sangrá (2012) discurren que los indicadores son necesarios en un proceso de planeación, pues los indicadores son las alertas sobre cómo se avanza y los logros encontrados. Unos indicadores bien establecidos son garantía para el logro de objetivos de un plan de apropiación de las TIC, adicionalmente, permiten la medición de logros. " ...se considera prioritario disponer de un sistema de indicadores para acometer un Plan de evaluación continua del proyecto. (Salinas, 2008, p.140). Sin estos indicadores no es posible monitorear y hacer los ajustes necesarios al plan que tenga la institución para el uso de las TIC.

Un $12.50 \%$ de los docentes responde que en su Institución educativa hay un manual de convivencia que contempla a las TIC y uso de internet, reglamento, cuidados y el manejo de situaciones de diversas índoles, mientras un $40 \%$ contestó que el manual de convivencia vislumbra a las TIC y el reglamento de uso de las salas, no avista cuidados y uso de internet. Un $27.50 \%$ contesta que el manual de convivencia no tiene nada sobre las TIC.

Tener un manual de convivencias que reconoce las normas para un uso adecuado, ético y responsable de la tecnología es un indicador de la importancia que reviste la tecnología para la institución educativa, para ello, Duque y Rojas (2012), examinan una serie de normas a tener en cuenta en este manual, las cuales se pueden expresar como competencias a tener en cuenta en la era digital:

Los usuarios:

"Exhiben comportamientos legales y éticos, en lo que concierne al empleo de la tecnología y de la información.

-Comprenden las implicaciones legales y éticas del uso de las licencias de software.

-Previene, advierte y hace comprender por el alumno las diversas situaciones de plagio o fraude en sus trabajos escolares para que no incurra en ellas.

-Promueven en la comunidad escolar el uso ético y legal de las aplicaciones informáticas e informaciones disponibles en sus diferentes formatos." (Duque \& Rojas, 2012, p.52).

\section{Gestión pedagógica:}

Los docentes responden en esta categoría sobre el uso pedagógico de la tecnología, va más allá de un uso instrumental del computador, con lo cual el estudiante no tiene inconveniente, de hecho, en esta se hace una reflexión sobre que se enseña, que se aprende y que se evalúa, adicionalmente, 
que motiva al estudiante a aprender, en especial usando las tecnologías, ello se logra mediante procesos de formación en estrategias pedagógicas y didácticas con el uso de tecnologías en el aula (Salinas, 2004), (Rojas et al, 2013). Con los cambios permanentes de la tecnología, los docentes deben estar en permanente formación y actualización, lo cual hará que la innovación en ellos se convierta en parte de su vida, innovar no debe ser una alternativa en un punto de tiempo, debe ser una forma de vida y cada vez que un profesor pone en prueba un nuevo diseño, ya está pensando cómo mejorarlo o adecuarlo para que cumpla de mejor manera su función, ya sea usando otra aplicación, lenguaje o lo que haya usado para generar su AHD (Rojas et al., 2013).

Los educadores que respondieron las preguntas, creen que la Institución no tiene espacios de reflexión en ella o participa en espacios fuera de esta, son conscientes de la importancia de la tecnología y admiten la importancia de los espacios donde estos temas se discuten y tratan de hacer presencia cuando pueden, pero no es una política Institucional. Ningún docente considero no conocer la importancia de las TIC en educación.

El 12,50\% de los encuestados opinan que la institución educativa tiene interés en la investigaciones sobre el uso y apropiación de TIC, sin embargo, la mayoría piensa que su institución no le da importancia, pero ellos han logrado hacer procesos de investigación sobre el tema dado que adelantan estudios de postgrado y sus trabajos o tesis terminan siendo sobre estos temas en sus mismas instituciones. Frente a esto, la UNESCO (2008) dice que "Desde esta perspectiva, los docentes son aprendices expertos y productores de conocimiento, permanentemente dedicados a la experimentación e innovación pedagógicas, para producir nuevo conocimiento sobre prácticas de enseñanza y aprendizaje" (UNESCO, 2008, p. 18). Siendo la investigación de los fenómenos académicos con las TIC que ocurren en la misma institución, un referente para mejorar los procesos institucionales y medir mejor esos indicadores de apropiación que son la guía para conocer cómo avanza el proceso.

El 15\% de los docentes supone que los procesos de formación son articulados con la institución educativa y están en un plan de trabajo, mientras que el $45 \%$ reflexiona que han participado de manera obligatoria en procesos de MINTIC y el MEN y un $30 \%$ lo ha hecho por su cuenta en estudios postgraduales y formaciones por cuenta propia. Tener establecido un modelo pedagógico que contemple a las TIC es muy importante, en especial si este se encuentra en el PEI o hace parte de los documentos formales Institucionales (Duque \& Rojas, 2012), también es importante que en estos documentos formales se establezca todo el trabajo conjunto que se realiza en clases y fuera de esta, basado en las AHD desde un DTP, lo cual pueda garantizar un valor agregado de las TIC al proceso educativo y su seguimiento (Coll, 2008) y (Rojas, et al. 2013).

\section{Conclusiones y recomendaciones}

El estudio permitió, desde la base teórica trabajada con los documentos referentes, entre ellos UNESCO, diseñar un instrumento que permitiera identificar desde la gestión administrativa, tecnológica y pedagógica los modelos a los cuales se ajusta la innovación educativa con las TIC de aquellos docentes que fueron seleccionados por sus trabajos para representar a sus instituciones en el Educa Digital Regional. Este instrumento no sólo contribuye al proceso de indagación sobre los modelos de innovación educativa con 
las TIC, sino que aporta al seguimiento y monitoreo del proceso en cualquier Institución educativa, midiendo su avance y desarrollo. Bates \& Sangrá consideran que "...una estructura de gobernanza coherente sirve de marco para la gestión eficaz de la tecnología para transformar la enseñanza y el aprendizaje en las instituciones" (2012, p.259).

Los resultados arrojados por la aplicación de los instrumentos, permitió conocer los modelos predominantes entre los docentes innovadores, los modelos que se presentan tienen elementos de gestión administrativa, tecnológica y pedagógica, estos también tienen características de varios modelos, como el llanero solitario de Bates (2004) o iniciación de Salinas (2004), el evolucionados, radical, colegiado y otros, sin embrago, la ubicación en un modelo específico tiene una fuerte tendencia hacia el de iniciación (Salinas, 2004) o el llanero solitario (Bates, 2004) pues los docentes no cuentan con equipos de trabajo, normalmente trabajan solos y la institución educativa simplemente apoya poco algunas y moderadamente otras, pero sin tener recursos apropiados para el desarrollo de un modelos de educación apoyada en tecnologías y siendo los docentes la excepción a la regla, no el modelo común entre los docentes.

El modelo general encontrado entre los encuestados, es un modelo donde el docente normalmente trabaja de manera individual, adicionalmente, no hay un grupo de expertos que lo acompañe y lo apoye, lo más cercano a este equipo, es el gestor enviado por CPE (Computadores Para Educar) que lo acompaña y le ayuda a mejorar sus recursos educativos cuyo producto es una AHD. En cuanto a la gestión directiva, se hace notar que no es para la dirección institucional prioritario los asuntos sobre las TIC, por ello, las recomendaciones anteriores pueden ayudar a dar un salto cualitativo y cuantitativo en este aspecto, así mismo, el contemplar en el PEI, manual de convivencia y plan estratégico de TIC (Plan estratégico de TIC o Temáticas) los temas relacionados con las TIC, los usos pedagógicos y estrategias didácticas que puede enriquecer el proceso de enseñanza y aprendizaje son fundamentales, Por ello, deben establecer unos indicadores a los que se le pueda hacer seguimiento.

\section{Otras consideraciones:}

Las Instituciones deben tener unos indicadores claros de uso de su dotación tecnológica, la cual le permita definir qué tan usados estas siendo estos recursos, lo que permitirá responder si requieren más equipos o aumentar la demanda de estos; es posible que se encuentren sub utilizados y se establezcan estrategias de uso con la comunidad o los mismos docentes y estudiantes.

El manual de convivencia de las Instituciones educativas debe contemplar las TIC y tener la reglamentación de su uso, cuidado, deberes y derechos de los usuarios, en este se deben contemplar los equipos de escritorio, portátiles y dispositivos móviles, manejo de los sitios no permitidos por la Institución, matoneo cibernético y horarios de trabajo en estos. Se encuentra que en la mayoría de Instituciones, esto no se tiene presente en el manual de convivencia.

Es importante que las Instituciones traten de establecer espacios, así sea una vez al año, donde las TIC y su uso en educación sean el plato fuerte, este espacio debe estar contemplado en los documentos institucionales.

Las investigaciones que la Institución apoye o las que realicen los docentes por su cuenta en sus estudios de postgrado deben 
ser objeto de socializaciones al interior de la Institución, con el colectivo docente y directivo, abrir espacios de transferencias de conocimientos para que estos docentes puedan multiplicar sus experiencias.

Los procesos de formación deben ser de alguna manera articulados por la Institución educativa, ya que esta tiene unas necesidades y lo mejor que puede ocurrir es que las necesidades de formación de los docentes se articulen con los procesos de formación de sus docentes.

En este momento se considera, según los resultados, que el modelo de gestión para innovar con las TIC en el aula sigue siendo el modelo de iniciación, con algunos elementos adicionales, aun así, el docente sigue siendo, como lo denomina Tony Bates, un "llanero solitario" (Bates, 2004).

\section{Referencia Bibliográfica}

Barceló L, María. (2001). Hacia una economía del conocimiento. Editorial Pricewaterhouse Coopers. Madrid. 210 p.

Bates, Antony (Tony). (2004) La transformación de las Universidades: a traves de las TIC: Discursos y prácticas. Barcelona, UOC.

Bringué, X., Sádaba, C. C., \& Tolsá-Caballero, J. (2011). La generación interactiva en Iberoamérica 2010. Niños y adolescentes ante las pantallas. Colección Fundación Telefónica, Ariel. Barcelona.

Cajiao, Francisco y otros. (1998). El largo y sorprendente viaje de los Pleyades. MEN. Fundación FES. Colombia.

Cajiao. Francisco y PARODI Martha. (1977). Proyecto Nautilus. El espíritu científico de la escuela. Colciencias. FES. Colombia.

Casassus, J. (1999). Marcos conceptuales para el análisis de los cambios en la gestión de los sistemas educativos. La gestión: en búsqueda de sujeto. Seminario internacional-Reformas de la gestión de los sistemas educativos en la década de los noventa-Santiago de Chile, 1314.

Casassus, J. (2000). "Problemas de la gestión educativa en America Latina" UNESCO, Chile.

Chabolla Romero, J. Manuel. (2001). Un proyecto de docencia: para las instituciones de educación superior (IES) en México. Plaza y Valdés. México.

Cilleruelo, E. (2010). Compendio de definiciones del concepto «Innovación» realizadas por autores relevantes: diseño híbrido actualizado del concepto. Dirección y Organización, (34), 91-98.

Coll, Cesar. (2008). Psicología de la Educación Virtual. Madrid. Ed. Morata.

Coll, C. (2007) TIC y prácticas educativas: realidades y expectativas. Ponencia magistral presentada en la XXII semana Monográfica de Educación. Fundación Santillana. Madrid. Disponible en: http://www.oei.es/tic/santillana/ coll.pdf

Coll, C. y Monereo, C. (Eds.) (2008) Psicología de la Educación Virtual. Aprender y enseñar con las Tecnologías de la información y la comunicación. Madrid. Morata.

Coll, C., Mauri, T. y Onrubia, J. (2008). La utilización de las Tecnologías de la Información y la Comunicación: Del diseño Tecnopedagógico a las prácticas de uso. En: Coll, C y Monereo, C. (Eds.) Psicología de la Educación Virtual. Aprender y enseñar con las Tecnologías de la información y la comunicación. Ediciones Morata : Madrid. (74-103)

Coll, C.,Mauri T. y Onrubia, J. (2008). El análisis de los procesos de enseñanza $\mathrm{y}$ aprendizaje mediados por las TIC: una perspectiva constructivista. En:

CPE. (2012). Convocatoria de formación y acompañamiento docente en la región 3. CPE: Bogotá.

Deusto, (2004). Hardvard Business essencial: Desarrollar la gestión de la creatividad y de la innovación. Editorial Planeta DeAgostini. Barcelona. 
Duque, Eduardo \& Rojas, Jorge. (2012). La formación docente en TIC y su uso en los proyectos pedagógicos de aula. Tesis de maestría. UTP: Pereira

Dussel, I. (2011). Aprender y enseñar en la cultura digital. Documento Básico del VII Foro. Buenos Aires: Santillana.

Galeana. De La O. Lourdes. (2011). Aprendizaje Basado en Proyectos. Disponible en: http:// ceupromed.ucol.mx/revista/PdfArt/1/27.pdf Consultado: (03/02/2013).

Gutiérrez G. Martha y Zapata. S. María Teresa. (2009). Los proyectos de aula. Una estrategia pedagógica para la educación. Colombia. Editorial Rocca.

Merli, Giorgio. (1997). La gestión eficaz, Ediciones Díaz de santos, Madrid. 219 P.

Moore, M. Kearsley. G.(1996). Distance Education, a Systems View. Wadsworth Publishing Company, California, USA.

Nieto Nieto, J. (2012). Y tú...i Innovas o abdicas?. Valencia. Editorial Universitat Politécnica de València.

Rojas, Jorge, Amador, Francisco, Sánchez, Héctor \& Duque, Eduardo. (2013). Las Ayudas Hipermediales Dinámicas (AHD) en los proyectos de aula en TIC. Otra forma de enseñar y aprender conjuntamente. Computadores Para Educar - Universidad Tecnológica de Pereira. Estrategia integral de formación y acceso. Pereira - Colombia.

Ruas, Yogel. (2011). Impacto de la formación docente en TIC en el departamento de Risaralda. Tesis de maestría. UTEM: Chile.

Salinas, Jesús. (2004). Innovación docente y uso de las TIC en la enseñanza universitaria, Revista Universidad y sociedad del conocimiento, V.1 N 1. UOC.2004.

Schumpeter, J. (1935). Análisis del cambio económico. The Review of Economics Statistics, vol. XVII, nº 4, pp. 2-10.
Unesco, (2005). Hacia las sociedades del conocimiento, Unesco, Francia, 237 $\mathrm{p}$ Disponible en: [http://unesdoc.unesco. org/images/0014/001419/141908s.pdf] [consultado: 25/03/2013] 